\title{
Single-Port 5T SRAM Cell with Improved Write-Ability and Reduced Standby Leakage Current
}

\author{
Chien-Cheng Yu and Ming-Chuen Shiau
}

\begin{abstract}
In this paper, a novel single-port five-transistor (5T) Static Random Access Memory (SRAM) cell and associated read/write assist is proposed. Amongst them, a voltage level conversion circuit is to provide a voltage of the respective connected word line to be lower than or equal to the power supply voltage VDD, as such the read/write-ability of the cell can be improved. Furthermore, a voltage control circuit is coupled to the sources corresponding to the driver transistors of each row memory cells. This configuration is aimed to control the source voltages of driver transistors under different operating modes. In addition, a pre-charging circuit is design to pull up the bit line BL of a selected column to the voltage VDD before the read or write operation. Finally, with the standby start-up circuit design, the memory cell can rapidly switch to the standby mode, and thereby reduce leakage current in standby. In particular, the paper introduce a two-phase reading mechanism to improve the reading speed, and thus to avoid unnecessary power consumption. Furthermore, by using the voltage level conversion circuit to pull the voltage of the signal WLC in a selected row cells lower than the power supply voltage VDD by a threshold voltage during a read operation, thereby to reduce the half-selected cells disturbance.
\end{abstract}

Index Terms-Read/write assist circuitry, standby start-up circuit, static random access memory, voltage level conversion circuit, voltage control circuit.

\section{INTRODUCTION}

Memory plays an indispensable role in computer industries. Usually, a memory is classified into a DRAM (dynamic random access memory) and an SRAM (static random access memory) according it's respective data storage capability. DRAM is advantageous for its small size, but requires periodic refresh to prevent data loss due to current leakage. SRAM requires no refreshing and will maintain its information as long as it has sufficient power supplied. This is due to the fact that the SRAM cell includes flip-flop circuitry internally that does not require refreshing, but occupies a large chip area.

An SRAM cell has three modes of operation, namely read, write and standby [1]. The data stored in the cells may be corrupted when the cells are read. This problem arises from the fact that a higher voltage on the bit line is coupled to a lower voltage in the cell, causing the bit line voltage to drop and the cell voltage to rise. Further, a concern associated with the write operation is that it is relatively difficult to write a

Manuscript received August. 5, 2016; revised January 13, 2017.

Chien-Cheng $\mathrm{Yu}$ and Ming-Chuen Shiau are with the Department of Electronic Engineering, Hsiuping University of Science and Technology, Taichung, Taiwan (e-mail: jenkenyu@gmail.com). logical ' 1 ' to the cell if the cell currently stores a logical ' 0 '. Accordingly, the SRAM cell should provide less likely to be corrupted when the cell is read and more reliable when the cell is written [2]. As integrated circuits become smaller and denser and as power consumption specifications for battery powered integrated circuits decrease, along with power supply voltages, the present SRAM cell designs are increasingly inefficient in both silicon area used and power consumed.

Memories take up $80 \%$ of the die area in high performance processors [3]. Therefore, there is a crucial need for a low leakage and highly robust SRAM design. Leakage current from a memory cell can cause unnecessary power consumption, especially during a standby mode. Recent research has shown that the leakage current will become even more than the dynamic current in the overall power consumption [4]. Typically, there are three major sources of leakage in a MOS transistor, namely subthreshold leakage, gate leakage, and reverse bias junction leakage [5]. Amongst them, the Gate-Induced drain leakage (GIDL) is an unwanted short-channel effect that occurs at higher drain biases in an overdriven off state of a MOS transistor. However, the Drain-induced barrier lowering (DIBL) is a short-channel effect in MOS transistors referring originally to a reduction of threshold voltage of the transistor at higher drain voltages. With scaling down of the MOS transistor, each of the leakage sources may increase accordingly, thus resulting in the increase of the total leakage current. In particular, as the CMOS technology scales down to $90 \mathrm{~nm}$ and below, the power consumption caused by leakage currents is becoming a significant part of the global power consumption [6]. Therefore, it would clearly be desirable to provide a design for an SRAM cell that has less leakage current than traditional designs when the cell in standby.

The remainder of this paper is organized as follows. Section 2 presents a brief description of standard 6T and 5T SRAM cell topologies. The proposed single-port 5T SRAM cell with integrated read/write assist is described in Section 3. The simulation results of the proposed 5T SRAM cell are discussed in Section 4. Last section is a conclusion and summary for the paper.

\section{EXISTING 6T AND 5T SRAM CELL TOPOLOGIES}

The standard 6T SRAM is built up of two cross-coupled inverters (INV-1 and INV-2) and two access transistors (MA1 and MA2), connecting the cell to the bit lines (BL and BLB), as shown in Fig. 1 [7]. The pair of cross-coupled inverters is formed by a pair of load transistors (MP1 and MP2) and a pair of driver transistors (MN1 and MN2) that are 
stronger than the access transistors. More specifically, the cross-coupled inverters of the memory cell have two storage nodes A and B functioning to store either logic ' 1 ' or logic ' 0 '. The gates of the access transistors are connected to a word line WL, and a rising transition on the word line to assert the access transistors during a read or a write operation. While, at the end of the read and write operations, the word line WL is de-asserted to allow the cross-coupled inverters to function normally and hold the logic state of the storage nodes.

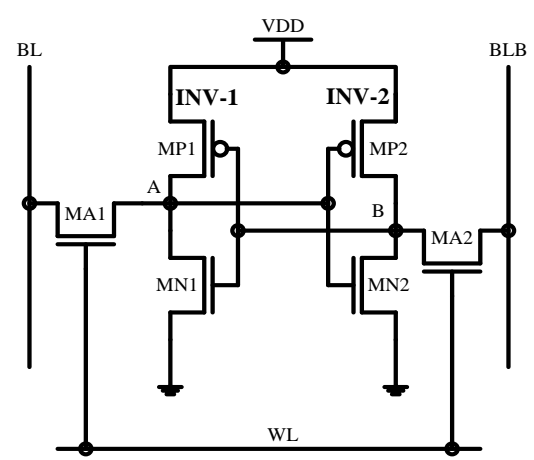

Fig. 1. Circuit diagram of standard 6T SRAM cell.

A concern associated with the read operation is that because of the back-to-back connection of cross-coupled inverters, a regenerative action develops and node $\mathrm{A}$ is pulled high resulting in the destruction of contents in the bit cell. Especially, when a logical ' 0 ' is stored initially, the voltage rising in the cell may corrupt the data stored. Therefore, it is desirable to keep the voltage at the storage node which has a logical ' 0 ' stored from rising above the trip-voltage of the inverter. To provide a non-destructive read operation, the cell ratio (CR) was conventionally varied from 1 to 2.5 [2], where the $\mathrm{W} / \mathrm{L}$ ratio of the driver transistor to the access transistor is referred to as the cell ratio. On the contrary, for a successful write operation, it may be necessary that the access transistors should be very conductive to force the cross-coupled inverters to change its equilibrium condition. However, the access transistor should have a reduced conductivity for good stability in reading and standby operations. These two requirements impose contradicting requirements on cell transistor sizing. To improve the read-ability of an SRAM cell, cell ratio can be increased, while a lower pull-up ratio is desirable to improve the cell write-ability, where the ratio of the load transistor to the access transistor is referred to as the pull-up ratio (PR).

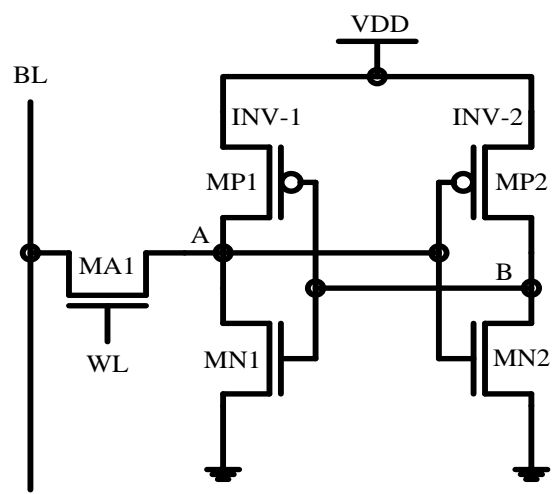

Fig. 2. Circuit diagram of traditional 5T SRAM cell.
Fig. 2 is a circuit diagram of a traditional 5T SRAM cell [8]. As shown in Fig. 2, the access transistor MA2 and bit line BLB in Fig. 1 have been removed to provide a five-transistor configuration. The removal of such access transistor allows for an area savings up to $20-30 \%$ compared to the standard 6T SRAM cell, while its power consumption is substantially reduced by one half [9]. Although the traditional 5T SRAM cells offer such significant reductions in power consumption, a serious drawback is presented in that it is difficult to write ' 1 ' to the cells. In detail, when the bit line BL is set high and the word line WL is asserted, the transistors MA1 and MN1 fight one another. To guarantee a correct write operation will occur, it is important to note that the storage node A must be pulled up (or down) above (or below) the trip-voltage of INV-2 within the word line WL is logic high, otherwise a write failure will occur. In more detail, writing a logical ' 1 ' to a cell when initially a logical ' 0 ' is stored, the low storage node $\mathrm{A}$ of the cell must be pulled up by the pre-charged bit line BL above the trip-voltage of INV-2.

In order to resolve the write ' 1 ' issue of the traditional $5 T$ SRAM cells, several techniques have been developed. Some of these techniques rely on boosted word line voltage [10]-[12], reducing the supply voltage VDD [8]-[9], [13]-[14], sizing cell transistors [15]-[17], reduced bit line voltage [18]-[19], and raising the source voltage $V_{S S}$ [20]-[22]. However, each of these techniques may cause a reduction in the drive current of the transistors and in the operating speed of the cell, or has increased memory cell area and a degradation in the manufacturing accuracy, or requires generation of a voltage above the operating voltage, or requires a more complicated circuit design and more complicated device process. Hence, there is a need for an effective technique to improve the write-ability of 5T SRAM cells which suffer from inability to write ' 1 '.

\section{THE PROPOSED 5T SRAM CELL}

\section{A. The Proposed 5T SRAM Cell Configuration}

The proposed 5T SRAM cell with read/write assist circuitries is shown in Fig. 3. The read/write assist including a level conversion circuit, a pre-charging circuit, a standby start-up circuit and a voltage control circuit. Amongst them, the level conversion circuit is to provide a voltage of the selected word line when the respective word lines are in an active state. Unlike the traditional 5T SRAM cell in Fig. 2, a pull-down word line voltage VDD- $\mathrm{V}_{\mathrm{TN} 51}$ is applied to the word line control signal WLC of the selected row cells so as to improve the cell read- and write-ability. In detail, the level conversion circuit weakens the access transistor N13 such that the voltage drop across the transistor N13 increases and the voltage drop between the transistor N13 and the driver transistor N11 reduces, thereby increasing the read-ability. It is worth noting that the word line control signal WLC of the selected cell is provided a voltage VDD- $\mathrm{V}_{\mathrm{TN} 51}$ during a read operation, wherein $\mathrm{V}_{\mathrm{TN5} 1}$ is the threshold voltage of the transistor N51. However, the power supply voltage VDD is supplied during a write operation. In this conversion circuit, the read signal $\mathrm{R}$ and the inverse write signal $/ \mathrm{W}$ can be achieved from the memory read/write control pin. When the 
signal $\mathrm{R}$ is at logic high, it indicates that the cell is in a read operation, and however the inverse write signal / $\mathrm{W}$ is at logic low indicates a write operation. And, the inverse signal of the $\mathrm{read} /$ write control pin is same as the inverse read/write control signal /RW. The voltage level of the signal WLCs under different operating modes is shown in Table I. It is worth noting that the paper introducing a two-phase reading mechanism to improve the reading speed, and thus to avoid unnecessary power consumption. Furthermore, by using the voltage level conversion circuit to pull the voltage of the signal WLC in a selected row cells down to VDD- $\mathrm{V}_{\mathrm{TN} 51}$ during a read operation, thereby to reduce the half-selected cells disturbance.

TABLE I: Voltage LeVel of THE SignAL WLC Under DifFerent OPERATING MODES

\begin{tabular}{ccccccc}
\hline Cells & $\mathrm{WL}$ & $\mathrm{R}$ & $\bar{W}$ & $\overline{R W}$ & $\mathrm{WLC}$ & Mode \\
\hline $\begin{array}{c}\text { Selected row } \\
\text { cells }\end{array}$ & $\mathrm{V}_{\mathrm{DD}}$ & $\mathrm{V}_{\mathrm{DD}}$ & $\mathrm{V}_{\mathrm{DD}}$ & $\mathrm{V}_{\mathrm{DD}}$ & $\mathrm{V}_{\mathrm{DD}}-\mathrm{V}_{\mathrm{TM} 51}$ & Read \\
\hline $\begin{array}{c}\text { Non-selected } \\
\text { row cells }\end{array}$ & 0 & $\mathrm{~V}_{\mathrm{DD}}$ & $\mathrm{V}_{\mathrm{DD}}$ & $\mathrm{V}_{\mathrm{DD}}$ & 0 & Read \\
\hline $\begin{array}{c}\text { Selected row } \\
\text { cells }\end{array}$ & $\mathrm{V}_{\mathrm{DD}}$ & 0 & 0 & $\mathrm{~V}_{\mathrm{DD}}$ & $\mathrm{V}_{\mathrm{DD}}$ & Write \\
\hline $\begin{array}{c}\text { Non-selected } \\
\text { row cells }\end{array}$ & 0 & 0 & 0 & $\mathrm{~V}_{\mathrm{DD}}$ & 0 & Write \\
\hline Each cell & 0 & 0 & $\mathrm{~V}_{\mathrm{DD}}$ & 0 & 0 & Non-access \\
\hline
\end{tabular}

In addition, there is a pre-charging circuit that connected to the bit line $\mathrm{BL}$ in each column. The function of the pre-charging circuit is to pull up the bit line BL of a selected column to VDD before the read or write operation. Furthermore, the standby start-up circuit design is to enable the SRAM cell to quickly switch to the standby mode, and thus effectively enhance the standby performance. Refer to Fig. 3, when the write control signal WC is at logic low, the voltage of node $C$ will be equal to that of the inverse standby control signal /S. On the contrary, when the write control signal WC is at logic high, the voltage of node $\mathrm{C}$ will be equal to that of the voltage of the control signal S. Thereby, in a standby operation, to effectively avoid the error writing caused by unexpected factors. Wherein, in the non-read mode, the voltage of the read control signal RC is set to the voltage RGND to prevent the leakage current caused by the transistor N24 in the non-read mode. Furthermore, during the initial period in standby, the standby start-up circuit is designed to rapidly charge the parasitic capacitance of the node $\mathrm{L} 1$ to the voltage $\mathrm{V}_{\mathrm{TN} 23}$.

Particularly, in this paper a two-phase read mechanism is introduced to speed up the reading speed and thus to avoid unnecessary power consumption during a read operation. Furthermore, during a write operation, the voltage $\mathrm{V}_{\mathrm{L} 1}$ is set to $\mathrm{V}_{\mathrm{GS}(\mathrm{N} 23)}$ and that of node $\mathrm{L} 2\left(\mathrm{~V}_{\mathrm{L} 2}\right)$ is set to the ground voltage, wherein $\mathrm{V}_{\mathrm{GS}(\mathrm{N} 23)}$ is the threshold voltage of the transistor N23. Thus, the issue concerning the difficulty of writing ' 1 ' can be resolved. In addition, during a standby operation, both $\mathrm{V}_{\mathrm{L} 1}$ and $\mathrm{V}_{\mathrm{L} 2}$ are set to $\mathrm{V}_{\mathrm{GS} \text { (N23) }}$ to reduce the leakage current in standby. Table II summaries the operating conditions under different operating modes. In Table II, the write control signal WC can be achieved by performing the AND operation on the write signal $\mathrm{W}$ and its corresponding word line signal WL. And, the read control signal RC can be achieved by performing the AND operation on the read signal R and its corresponding word line signal WL.

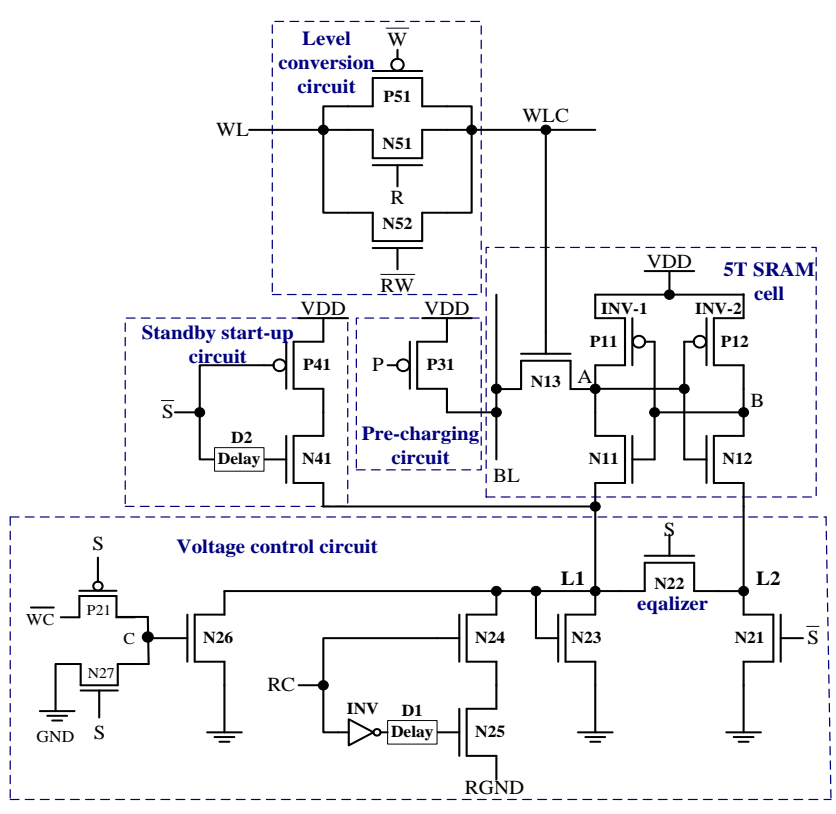

Fig. 3. Circuit diagram of the proposed 5T SRAM cell.

TABLE II: THE OPERATING CONDITIONS UNDER DIFFERENT OPERATING

\begin{tabular}{lccccc}
\multicolumn{5}{c}{ MODES } \\
\hline RC & WC & $\mathrm{S}$ & $\mathrm{V}_{\mathrm{L} 1}$ & $\mathrm{~V}_{\mathrm{L} 2}$ & mode \\
\hline RGND & 0 & 0 & $\mathrm{~V}_{\mathrm{GS}(\mathrm{N} 23)}$ & 0 & write \\
\hline $\mathrm{V}_{\mathrm{DD}}$ & 0 & 0 & $\begin{array}{c}\text { RGND (1st stage) } \\
\text { (2nd stage) }\end{array}$ & 0 & read \\
\hline RGND & 0 & $\mathrm{~V}_{\mathrm{DD}}$ & $\mathrm{V}_{\mathrm{GS}(\mathrm{N} 23)}$ & $\mathrm{V}_{\mathrm{GS}(\mathrm{N} 23)}$ & standby \\
\hline RGND & 0 & 0 & 0 & 0 & hold \\
\hline
\end{tabular}

An advantage of the proposed design over the traditional 5T SRAM cell is that it is unnecessary to boost the write line signal above VDD to speed up the read operation. Furthermore, this design has the additional advantage of increased current through the driver transistor during a read operation, and consequently lower read delay.

\section{B. Write Operation}

Refer to Fig. 3, prior to the write operation is performed, the write control signal WC is at logic low, the transistor P21 is turned on and the transistor N27 is turned off. Thereby, the node $\mathrm{C}$ is at logic high and thus to turn on the transistors N26, as such the voltage $\mathrm{V}_{\mathrm{L} 1}$ is pulled down to the ground voltage. However, during the write operation, the signal WC is at logic high, the transistors P21 is turned off and the transistor $\mathrm{N} 27$ is turned on. Subsequently, the node C is at logic low and thus to turn off the transistors N26, as such the voltage $\mathrm{V}_{\mathrm{L} 1}$ is set to $\mathrm{V}_{\mathrm{GS}(\mathrm{N} 23)}$. Thus, the issue concerning the difficulty of writing ' 1 ' can be resolved. Fig. 4 shows the simplified circuit diagram during the write operation.

The transients associated with a writing operation are detailed described below. Firstly, let us consider the write ' 0 ' operation. Prior to the write ' 0 ' operation, the voltage $\mathrm{V}_{\mathrm{BL}}$ and signal WLC are at logic low. During the write ' 0 ' operation, if a ' 0 ' is stored previously, the signal WLC transitions from a logic low to a logic high. As the signal WLC exceeds the threshold voltage of transistor N13 $\left(\mathrm{V}_{\mathrm{TN} 13}\right)$, transistor N13 is turned on. Subsequently, owing to the fact voltage $\mathrm{V}_{\mathrm{BL}}$ is at logic low, the voltage $\mathrm{V}_{\mathrm{A}}$ remains at the 
ground voltage. On the other hand, if a logical ' 1 ' is stored previously, the signal WLC transitions from a logic low to a logic high. As the signal WLC exceeds the threshold voltage $\mathrm{V}_{\mathrm{TN13}}$, transistor N13 is turned on. Subsequently, owing to the fact voltage $\mathrm{V}_{\mathrm{BL}}$ is at logic low, the node $\mathrm{A}$ and node $\mathrm{L} 1$ will be discharged to ground until the end of the write ' 0 ' operation.

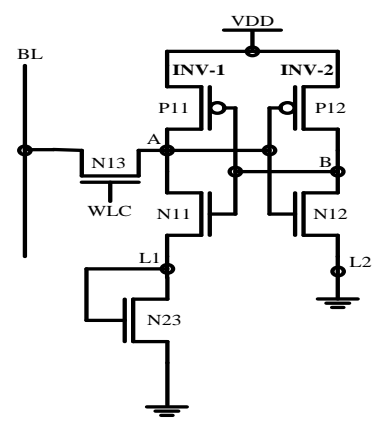

Fig. 4. Simplified circuit diagram during the write operation.

Secondly, consider the write ' 1 ' operation. Prior to the write ' 1 ' operation, the signal WLC is at logic low and the voltage $\mathrm{V}_{\mathrm{BL}}$ is at logic high. During the write ' 1 ' operation, if a ' 1 ' is stored previously, the signal WLC transitions from a logic low to a logic high. As the signal WLC exceeds the threshold voltage $\mathrm{V}_{\mathrm{TN} 13}$, transistor $\mathrm{N} 13$ is turned on. Subsequently, owing to the fact voltage $\mathrm{V}_{\mathrm{BL}}$ is at logic high and transistor $\mathrm{P} 11$ remains on, the voltage $\mathrm{V}_{\mathrm{A}}$ will remain at the power supply voltage VDD until the end of the write operation. On the other hand, if a ' 0 ' is stored previously, the signal WLC transitions from a logic low to a logic high. Subsequently, with the increase of the signal WLC, the voltage $\mathrm{V}_{\mathrm{A}}$ will rise. As the signal WLC exceeds the threshold voltage $\mathrm{V}_{\mathrm{TN} 13}$, transistor $\mathrm{N} 13$ is turned on. Subsequently, owing to the fact voltage $\mathrm{V}_{\mathrm{BL}}$ is at logic high and transistor $\mathrm{N} 11$ remains on, and the voltage $\mathrm{V}_{\mathrm{B}}$ remains at a voltage close to the power supply voltage VDD, the transistor P11 remains off. For a successful write operation, it is desirable to pulling down the voltage $\mathrm{V}_{\mathrm{A}}$ ( or $\mathrm{V}_{\mathrm{B}}$ ) which has a stored value ' 1 ' below the trip-voltage of the inverter. Meanwhile, the write initial transient voltage $V_{A w}$ of node $A$ must satisfy the following equation:

$$
V_{A W}=V_{D D} \times \frac{R_{N 11}+R_{N 23}}{R_{N 11}+R_{N 13}+R_{N 23}}>V_{T N 12}
$$

where, $\mathrm{V}_{\mathrm{TN} 12}$ is the threshold voltage of the transistor N12, $\mathrm{R}_{\mathrm{N} 11}, \mathrm{R}_{\mathrm{N} 13}$ and $\mathrm{R}_{\mathrm{N} 23}$ are the on-resistance of transistors $\mathrm{N} 11$, $\mathrm{N} 13$ and N23, respectively. Consequently, the write ' 1 ' problem associated with the traditional 5T SRAM cell can be avoided.

Now, the transistor N13 is still in the saturation region and the transistor N11 in the triode region. Although $\mathrm{R}_{\mathrm{N} 13}$ may be greater than $\mathrm{R}_{\mathrm{N} 11}$, the NMOS diode $\mathrm{N} 23$ can provide a voltage $\mathrm{V}_{\mathrm{GS} \text { (N23) }}$ at node $\mathrm{L} 1$. As a result, the voltage $\mathrm{V}_{\mathrm{A}}$ will rise up due to the voltage division along the driver and access transistors. When the voltage exceeds a threshold, it causes the bit to flip due to regenerative feedback. Hence, the write ' 1 ' operation is completed. Consequently, the write ' 1 ' problem associated with the traditional 5T SRAM cell can be resolved. It is worth noting that the voltage $V_{L 1}$ is $V_{G S}$ (N23) when writing a logical ' 1 ' to a logical ' 0 ' is stored. After completing the write ' 1 ' operation, the voltage $\mathrm{V}_{\mathrm{L} 1}$ will be discharged to ground via transistor N26.

\section{Read Operation}

Fig. 5 shows the simplified circuit diagram during the read operation. Prior to initiating a read operation, the bit line BL is pre-charged to VDD, the standby start-up control signal S, the write control signal WC and the read control signal RC are at logic low, thereby the transistors P21 and N25 are turned on and the transistors N24 and N27 are turned off, as such the voltage of node $\mathrm{C}$ is at logic high and subsequently turn on the transistor N26. This leads to the voltage $\mathrm{V}_{\mathrm{L} 1}$ will be pulled down to ground.

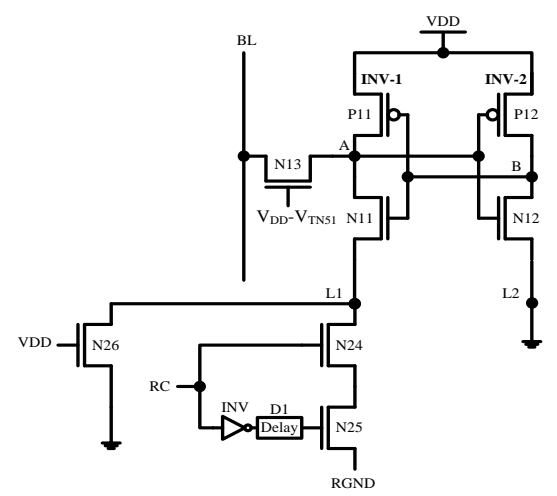

Fig. 5. Simplified circuit diagram during the read operation.

In this paper, a two-phase reading mechanism is introduced to increase the reading speed and thus to avoid unnecessary power consumption. In the first reading stage, the read control signal RC is at logic high, thereby transistor $\mathrm{N} 24$ is turned on. At this time, since transistor N25 would continue to conduct, the voltage $\mathrm{V}_{\mathrm{L} 1}$ will be pulled down to a negative voltage RGND as shown in Fig. 6. Under this circumstance, the negative voltage RGND can effectively improve the reading speed. However, in the second reading stage, the read control signal RC remains at logic high and the transistor $\mathrm{N} 24$ remains on. Consequently, $\mathrm{V}_{\mathrm{L} 1}$ is pulled up to the ground voltage due to the transistor N25 is turned off, and thus leads to reduce unnecessary power consumption. It is note that the two-phase time interval is measured as the time taken from a high on the read control signal RC to the state of the transistor N25 is turned off. This time interval can be adjusted by the falling time of the inverter INV and the delay time of the delay circuit D1. Furthermore, either in the first stage or the second stage, the transistor N26 is always on.

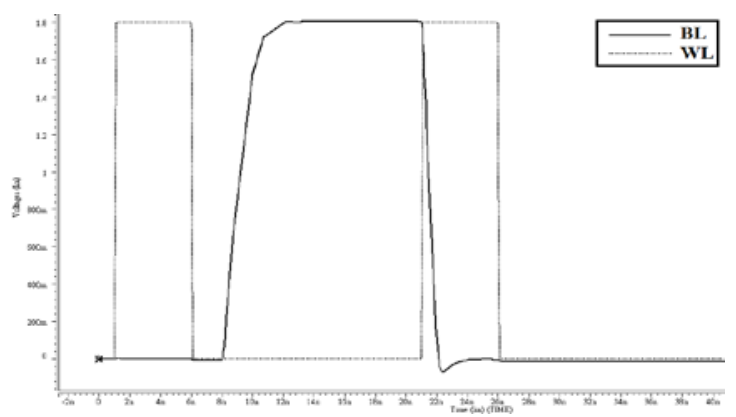

Fig. 6. Voltage level waveforms during a read operation.

The transients associated with a reading operation are 
detailed described below. Firstly, let us consider the read ' 1 ' operation. Before the read ' 1 ' operation occurs, the transistor $\mathrm{N} 11$ is off and the transistor $\mathrm{N} 12$ is on, and the voltage $\mathrm{V}_{\mathrm{A}}$ and the voltage $\mathrm{V}_{\mathrm{B}}$ are VDD and ground, respectively. And, the voltage $\mathrm{V}_{\mathrm{BL}}$ is equal to $\mathrm{VDD}$ due to the pre-charging circuit. During the read operation, since the voltage $\mathrm{V}_{\text {WLC }}$ is VDD- $V_{\mathrm{TN} 51}$, the transistor N13 is turned on. Thereby the voltage $\mathrm{V}_{\mathrm{BL}}$ can be effectively kept at VDD until the end of the read ' 1 ' operation. It is worth noting that due to the voltage RGND equaling to $\mathrm{V}_{\mathrm{L} 1}$, in order to effectively reduce the half-selected cell disturbance and effectively reduce the leakage current during the read ' 1 ' operation, the absolute value of the voltage RGND may be set to be smaller than the voltage $\mathrm{V}_{\mathrm{TN} 11}$ in reading ' 1 ', i.e.,

$$
|R G N D|<V_{T N 11}
$$

Where in, $|\mathrm{RGND}|$ denotes the absolute value of the voltage RGND and $\mathrm{V}_{\mathrm{TN} 11}$ is the threshold voltage of transistor N11.

Secondly, consider the read ' 0 ' operation. Before the read ' 0 ' operation is performed, the transistor N11 is on and the transistor N12 is off, and the voltage $V_{A}$ and the voltage $V_{B}$ are ground and VDD, respectively. And, the voltage $\mathrm{V}_{\mathrm{BL}}$ is equal to VDD due to the pre-charging circuit. During the read ' 0 ' operation, since the voltage $\mathrm{V}_{\mathrm{WLC}}$ is $\mathrm{VDD}-\mathrm{V}_{\mathrm{TN} 51}$, the transistor N13 is turned on. Meanwhile, the initial transient voltage $\mathrm{V}_{\mathrm{AR}}$ of the node $\mathrm{A}$ must satisfy the following equation:

$$
\begin{aligned}
V_{A R} & =V_{D D} \times \frac{R_{N 11}+\left(R_{N 24}+R_{N 25}\right) \| R_{N 26}}{R_{N 13}+R_{N 11}+\left(R_{N 24}+R_{N 25}\right) \| R_{N 26}} \\
& +R G N D \times \frac{\left(R_{N 11}+R_{N 13}\right) \| R_{N 26}}{R_{N 24}+R_{N 25}+\left(R_{N 11}+R_{N 13}\right) \| R_{N 26}} \times \frac{R_{N 13}}{R_{N 11}+R_{N 13}} \\
& <V_{T N 12}
\end{aligned}
$$

where in, $\mathrm{V}_{\mathrm{AR}}$ is the initial transient voltage of node $\mathrm{A}, \mathrm{V}_{\mathrm{TN} 12}$ is the threshold voltage of transistor N12, $R_{N 11}, R_{N 13}, R_{N 24}$, $\mathrm{R}_{\mathrm{N} 25}$ and $\mathrm{R}_{\mathrm{N} 26}$ are the on-resistance of transistors N11, N13, N24, N25 and N26, respectively.

It is worth noting that, the voltage RGND is designed to be lower than the ground voltage and its absolute value is designed to be smaller than the voltage $\mathrm{V}_{\mathrm{TN} 11}$. Furthermore, during the read ' 0 ' operation, the voltage $V_{\text {WLC }}$ is set to VDD- $\mathrm{V}_{\mathrm{TN} 51}$ as in the paper. As such this design can be increased $R_{\mathrm{N} 13}$ to satisfy the equation (3) and can reduce the half-selected cell disturbance in read ' 0 ' operation.

\section{Standby Operation}

Refer to Fig. 3, prior to the standby operation is performed, the inverse standby control signal / $\mathrm{S}$ is at logic high and thus the transistor P41 is turned off and the transistor N41 is turned on. And then, during the standby operation, the signal /S is at logic low to turn on the transistor P41 and to turn off the transistor N21. In addition, the high standby control signal $\mathrm{S}$ is to turn on the transistor N22 which acts as an equalizer. Consequently, with the conduction of the transistor $\mathrm{N} 22$, both the $\mathrm{V}_{\mathrm{L} 1}$ and $\mathrm{V}_{\mathrm{L} 2}$ are equal to the voltage $\mathrm{V}_{\mathrm{TN} 23}$ as shown in Fig. 7. It is worth mentioning that node L1 can be rapidly charged to $\mathrm{V}_{\mathrm{TN23}}$ at the initial period of the standby mode due to transistor N41 remains on, and thereby improving the standby efficiency. Note that the initial period is determined as the time taken from a low on the signal $\bar{S}$ to the state of transistor N41 is off. This time interval can be adjusted by the delay time of the delay circuit D2. It is worth noting that after the initial period of the standby mode, transistor N41 is turned off and no current flows. Fig. 8 shows the simplified schematic of the proposed design during the standby mode.

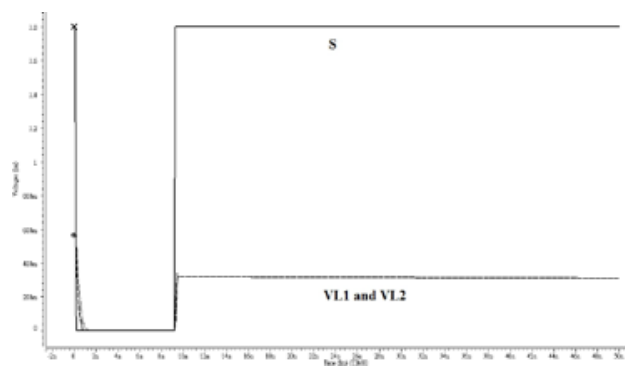

Fig. 7. Voltage level of node L1 and node L2 relative to the high standby control signal S.

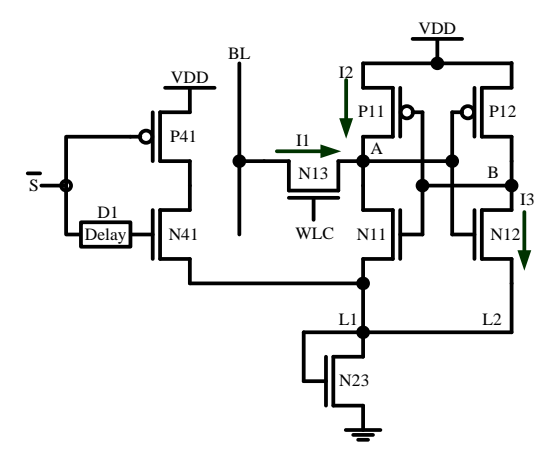

Fig. 8. Simplified circuit diagram during the standby operation.

\section{Simulation Results}

In this paper, the read operation is initiated by enabling the word line control signal WLC and connecting the pre-charged bit line BL to the storage node $\mathrm{A}$. If the word line control signal WLC is reduced, the voltage $\mathrm{V}_{\mathrm{A}}$ is also reduced during the read operation. In more detail, during the read operation, the word line control signal WLC in a selected row cell is set to a voltage VDD- $\mathrm{V}_{\mathrm{TN} 51}$. Thereby, the gate voltage of the access transistor N13 of the selected cell is reduced to decrease its current driving power, and thus leads to prevent data corruption in the read operation. On the contrary, during the write operation, the word line control signal WLC in a selected row cell is set to a power supply voltage VDD, and the word line control signal WLCs for the non-selected row cells are set to a ground voltage similar to that in a read operation so that a static noise margin in the read operation is improved, and a write margin is ensured. Apart from these modes, the word line control signal WLCs for the non-access cells are set to a ground voltage.

In the traditional 5T SRAM cell, the access transistor MA1 is less conductive than the driver transistor MN1, thereby making it more difficult to write a logical ' 1 ' to cell over a logical ' 0 ' is stored. Figure 9 shows the simulated waveform of a write ' 1 ' failure. To evaluate performance, different 
SRAM cell structures discussed in this paper were simulated using a 180nm CMOS technology. All simulations were carried out at nominal conditions: $\mathrm{VDD}=1.8 \mathrm{~V}$ and at room temperature. The simulated waveform of a successful writing in the proposed 5T SRAM cell is shown in Fig. 10. It is evident that the proposed 5T SRAM cell provides an efficient solution to the write ' 1 ' issue, that is, the proposed 5T SRAM cell enabling a logical ' 1 ' to be easily written to the SRAM cell, as compared to the traditional 5T SRAM cells.

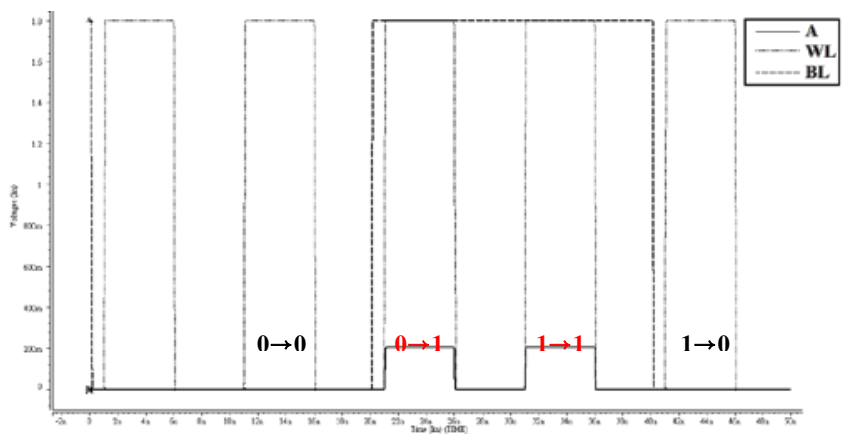

Fig. 9. Transient waveforms of a write failure in the traditional 5T SRAM cell.

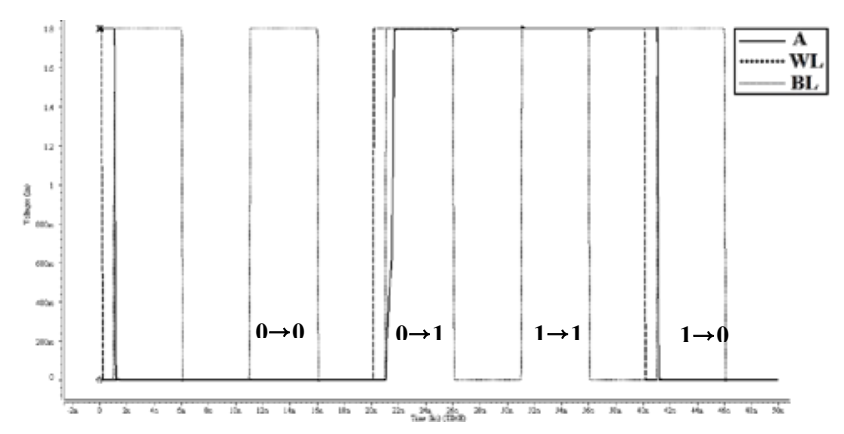

Fig. 10. Transient waveforms of a successful writing in the proposed 5T SRAM cell.

\begin{tabular}{cccc}
\multicolumn{4}{c}{ TABLE III: LEAKAGE CURRENT COMPARISON } \\
\hline $\begin{array}{c}\text { Corner } \\
\text { model }\end{array}$ & $\begin{array}{c}\text { Proposed 5T } \\
\text { SRAM } \\
(\mathrm{pA})\end{array}$ & $\begin{array}{c}\text { Standard 5T } \\
\text { SRAM } \\
(\mathrm{pA})\end{array}$ & $\begin{array}{c}\text { Improvement } \\
(\%)\end{array}$ \\
\hline TT & 11.6467 & 37.4557 & 68.9 \\
\hline SS & 9.917 & 12.0491 & 17.7 \\
\hline FF & 51.0658 & 590.8786 & 91.4 \\
\hline
\end{tabular}

Upon standby mode shown in Fig. 8, the voltage $V_{A}$ remains at $\mathrm{V}_{\mathrm{TN} 23}$, the voltage $\mathrm{V}_{\mathrm{WL}}$ is set to the ground voltage and $\mathrm{V}_{\mathrm{BL}}$ is set to VDD, respectively. Therefore, the gate-source voltage $\mathrm{V}_{\mathrm{GS}}$ of transistor $\mathrm{N} 13$ is negative. In contrast, the $\mathrm{V}_{\mathrm{GS}}$ of transistor MA1 in Fig. 1 is equal to zero. For NMOS transistors, according to the GIDL effect, the sub-threshold current at $\mathrm{V}_{\mathrm{GS}}=-0.1$ is approximately $1 \%$ of that at $\mathrm{V}_{\mathrm{GS}}=0$. Accordingly, the leakage current I1 flows through transistor N13 caused by the GIDL effect is much smaller than that of flowing through transistor MA1 in Fig. 1. Furthermore, the drain-source voltage $\mathrm{V}_{\mathrm{DS}}$ of transistor N13 is $\mathrm{VDD}-\mathrm{V}_{\mathrm{TN} 23}$, whereas the voltage $\mathrm{V}_{\mathrm{DS}}$ of transistor MA1 in Fig. 1 is VDD. According to the DIBL effect, the leakage current I1 flowing through transistor N13 is also less than that of flowing through transistor MA1 in Fig. 1. As a result, the leakage current flows through transistor N13 is much smaller than that of flowing through transistor MA1 in Fig. 1. Next, the source-drain voltage $\mathrm{V}_{\mathrm{SD}}$ of transistor P11 is
VDD- $\mathrm{V}_{\mathrm{TN} 23}$ in contrast to the $\mathrm{V}_{\mathrm{SD}}=\mathrm{VDD}$ of transistor MP1 in Fig. 1. According to the DIBL effect, the leakage current I2 flowing through transistor P11 will be less than that of flowing through transistor MP1 in Fig 1. Thus, the base-source voltage $\mathrm{V}_{\mathrm{BS}}$ of transistor $\mathrm{N} 12$ is negative, and the drain-source voltage $\mathrm{V}_{\mathrm{DS}}$ of transistor $\mathrm{N} 12$ is VDD- $\mathrm{V}_{\mathrm{TN} 23}$. On the contrary, the $\mathrm{V}_{\mathrm{BS}}$ of transistor MN2 in Fig. 1 is zero, and the $\mathrm{V}_{\mathrm{DS}}$ of transistor MN2 is VDD. According to the body effect and DIBL effect, the leakage current I3 flows through transistor N12 is much smaller than that of flowing through transistor MN2. From the above analysis, it can be seen that the proposed 5T single-port SRAM having a lower leakage current compared with the standard 6T SRAM. Table III shows a comparison of the simulated standby leakages of the traditional 5T SRAM cell and the proposed 5T SRAM cell using $180 \mathrm{~nm}$ CMOS technology. For each cell, the total leakage current flow, which is the sum of I1, I2 and I3 of all transistors are estimated.

As it can be seen from Table III, compared with the standard 5T SRAM cell in different corner models, the standby leakage current of the proposed design is significantly reduced $68.9 \%, 17.7 \%$ and $91.4 \%$, respectively.

\section{CONCLUSIONS}

This paper facilitates efficient data reading from and writing to an SRAM cell, particularly if a logical ' 0 ' stored in the cell is to be overwritten by a logical ' 1 '. With the level conversion circuit limits a word line voltage to a value lower than the supply voltage of the proposed 5T SRAM cell to improve the read-ability and write-ability is used in simulations. Simulation results for the proposed 5T cell design confirm that there is conspicuous improvement over the standard 5T SRAM cell while it allows writing ' 1 ' on the cell with read/write assist. In addition, with the proposed $\mathrm{read} /$ write assist leads to a $68.9 \%, 17.7 \%$ and $91.4 \%$ less standby leakage in different corner compared with the standard 6T SRAM cell.

\section{REFERENCES}

[1] N. H. E. Weste and D. M. Harris, "CMOS VLSI design: A circuits and systems perspective,” Addison Wesley, 2011.

[2] K. Itoh, VLSI Memory Chip Designs, Heidelberg, Springer-Verlag, 2001.

[3] M. Horowitz, "Scaling, power, and the future of MOS," in Proc. IEDM Tech. Dig., pp. 9-15, 2005.

[4] N. S. Kim et al., "Leakage current: Moore's law meets static POWER," IEEE J. Computer, vol. 36, no. 12, pp. 68-75, Dec. 2003.

[5] D. A. Hodges, H. G. Jackson, and R. A. Saleh, Analysis and Design of Digital Integrated Ciruits: In Deep Submicron Technology, 3rd ed., New York: McGraw-Hill Press, 2004.

[6] J. Rabaey, A. Chandrakasan, and B. Nikolic, Digital Integrated Circuits: A Designer Perspective, 2nd ed., Upper Saddle River, NJ: Prentice-Hall, 2003.

[7] H. Tran, "Demonstration of 5T SRAM and 6T dual-port RAM cell arrays,” in Proc. 1996 Symp. VLSI Circuits Dig. Tech. Papers, pp. 68-69, 1996

[8] I. Carlson, S. Andersson, S. Natarajan, and A. lvandpour, "A high density, low leakage, 5T SRAM for embedded caches,” in Proc. ESSCIRC 2004, pp. 215-218, Sept. 2004.

[9] S. Nalam and B. H. Calhoun, "5T SRAM with asymmetric sizing for improved read stability,” IEEE J. Solid-State Circuits, vol. 46, no. 10, pp. 2431-2442, Oct. 2011.

[10] S. Nalam, V. Chandra, C. Pietrzyk, R. Aitken, and B. Calhoun, "Asymmetric 6T SRAM with two-phase write and split bit line 
differential sensing for low voltage operation," in Proc. 11th Int. Symp. Quality Electronic Design (ISQED), pp. 139-146, Mar. 2010.

[11] Y. H. Chen et al., "A 0.6V 45nm adaptive dual-rail SRAM compiler circuit design for lower VDD_min VLSIs," in Proc. IEEE Symp. VLSI Circuits, pp. 210-211, June 2008.

[12] Y. Chung and S. H. Song, "Implementation of low-voltage static RAM with enhance data stability and circuit speed," Microelectronics Journal, vol. 40, no. 6, pp. 944-951, 2009.

[13] M. Yamaoka et al., "Low-power embedded SRAM modules with expanded margins for writing," in Proc. IEEE Int. Conf. Solid-State Circuits (ISSCC 2005), pp. 480-611, Feb. 2005.

[14] H. Pilo et al., "An SRAM design in 65nm and 45nm technology nodes featuring read and write-assist circuits to expand operating voltage," IEEE Symp. VLSI Circuits, pp. 15-16, 2006.

[15] K. J. O'Connor, "A source sensing technique applied to SRAM cells," IEEE J. Solid State Circuits, vol. 30, no. 4, pp. 500-511, Apr. 1995.

[16] M. H. Tu, J. Y Lin, M. C. Tsai, S. J. Jou, and C. T. Chuang, "Single-ended subthreshold SRAM with asymmetrical write/read-assist," IEEE Trans. Circuits and Systems- I: Regular Papers, vol. 57, no. 12, pp. 3039- 3047, Dec. 2010.

[17] M. C. Shiau, C. C. Yu, and K. T. Chen, "Single port SRAM having a lower power voltage in writing operation”, TW pat. I426514 B, Feb. 11 2014.

[18] D. P. Wang et al., "A 45nm dual-port SRAM with write and read capability enhancement at low voltage," in Proc. IEEE Int. SOC Conf., pp. 211-214, Sept. 2007.

[19] K. Nii et al., "A 45-nm single-port and dual-port SRAM family with robust read/write stabilizing circuitry under DVFS environment," in Proc. IEEE Symp. VLSI Circuits, pp. 212-213, June 2008.

[20] K. Kim, J. J. Kim, and C. T. Chuang, "Asymmetrical SRAM cells with enhanced read and write margins," in Proc. Int. Symp. VLSI Technology, Systems and Applications (VLSI-TSA), pp. 1-2, Apr. 2007.
[21] A. Bhavnagarwala et al., "Fluctuation limits \& scaling opportunities for CMOS SRAM cells," in Proc. IEEE Int. Electron Devices Meeting (IEDM 2005), pp. 659-662, Dec. 2005,

[22] H. S. Yang et al., "Scaling of 32nm low power SRAM with high-K metal gate," in Proc. IEEE Int. Electron Devices Meeting (IEDM 2008), pp. 1-4, Dec. 2008.

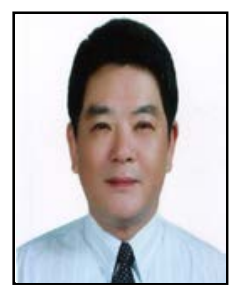

Chien-Cheng Yu received the B.S. and M.S. degrees from Feng Chia University and National Taiwan Normal University, respectively. He is currently an assistant professor with the Department of Electronic Engineering at Hsiuping University of Science and Technology, Taiwan. His current research interests include design and analysis of high-speed, low-power integrated circuits and low-voltage low-power embedded SRAM circuit design.

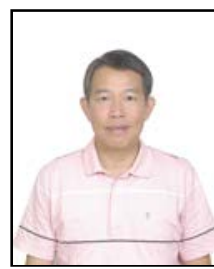

Ming-Chuen Shiau received the B.S., M.S. and Ph.D. degrees in electronic engineering from National Chiao Tung University, Hsinchu, Taiwan. He is currently a full professor with the Department of Electrical Engineering at Hsiuping University of Science and Technology, Taiwan. His current research interests include low-power digital circuit design, SRAM design and low-voltage embedded memory circuit design. 


\title{
Harmonic Suppression of High-Power UPS in Power Network
}

\author{
Lv Changzhi, Geng Anran, and Pan Jiaxing
}

\begin{abstract}
Rectifier is a necessary part of high-power UPS. But due to its nonlinear characteristics, serious input current harmonic pollution will be produced to the power network. In this paper, by theoretical analysis, calculation and simulation, the necessity of input current harmonic suppression of high-power UPS rectifier is proved. Sseveral harmonic control methods of input current are proposed according to two aspects: adding harmonic compensation device and improving rectifier architecture. Finally, by on-line test and parameter comparison, the feasibility of the scheme is verified.
\end{abstract}

Index Terms-High-power UPS, rectifier, input current, harmonic.

\section{INTRODUCTION}

With the development of power electronics technology, as an important equipment to ensure the stability and continuity of power supply, high-power UPS is widely used in power system, and comprehensively protects the hardware equipment, operating procedure and data transmission path of the network. AC/DC rectifier is a necessary part [1]. But due to the nonlinear characteristics, necessary analysises to the harmonic problem is very important for the selection and extension of the UPS rectifying before putting into use[2].

Under ideal conditions, after applying AC input sine wave voltage to the UPS, the input current waveform is changed into a non sinusoidal wave. In the course of running, the large number of current harmonics will generate reactive power, bring serious danger to power generation equipment, power supply equipment and power using equipment, cause a serious pollution on the power network and pose a threat to the safe operation of low voltage power supply system and all kinds of electric equipments [3].

In order to ensure the range of input current harmonic content of high-power and find an effective way to reduce it[4], in the paper, theoretical analysis, calculation and simulation of the input current harmonics are carried out. Several control methods are proposed to reducing the input current harmonic pollution. Actual tests or simulations are conducted to prove its feasibility, providing a theoretical basis for the harmonic suppression of high-power UPS in

Manuscript received October 10, 2016; revised January 13, 2017. This work is partially supported by Natural Science Foundation of China (61305129), China Postdoctoral Science Foundation (2012M521361), Shandong Province Natural Science Foundation (ZR2013CQ017), Domestic Visiting Scholars Program of Outstanding Young Teachers in Colleges and Universities in Shandong.

Lv Changzhi, Geng Anran and Pan Jiaxing are with Colege of Electrical Engineering and Automation, Shandong University of Science and Technology, Shandong, Qingdao 266590 China (e-mail: lvchangzhi@126.com,18765275951@163.com, hljpjx@126.com.) practical application.

\section{HARMONIC ANALYSIS}

\section{A. Theoretical Analyseie}

6 pulse rectifier is a common rectifying device of high-power UPS, which has a full bridge rectifier consisting of six thyristo and is controlled by six thyristor respectively. Its schematic diagram is shown in Fig. 1.

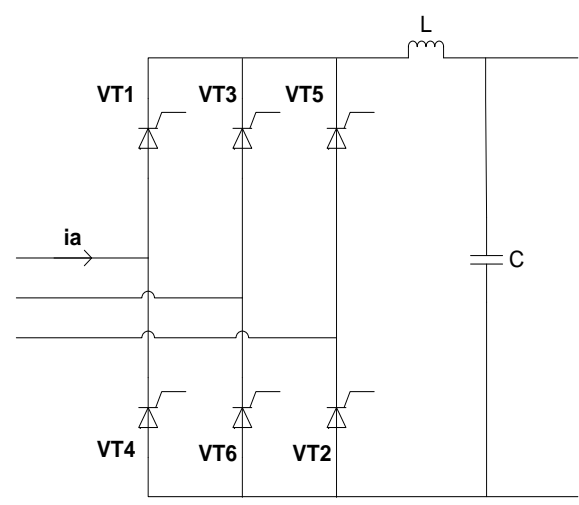

Fig. 1. 6 pulse rectifier.

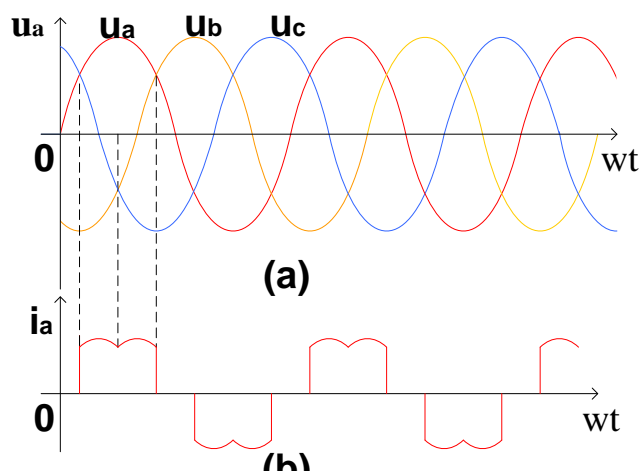

(b)

Fig. 2. 6 pulse rectifier waveform (a) AC voltage (b) A phase current.

Ignore phase change process and current pulse and the effect of the harmonic of power supply, assumed that the AC voltage is pure sine wave, the AC side reactance is zero and trigger delay angle $\alpha=0.6$ pulse rectifier (a) AC side voltage waveform (b) A phase current waveform is shown in Fig. 2. While the input sine wave voltage flows through the rectifier bridge, the input current is not a sine wave but a saddle shaped wave, its positive or negative half cycle of each wide is $120^{\circ}$ and the front difference is $180^{\circ}$ [5].

\section{B. Harmonic Calculation and Total Harmonic Distortion}

Taking A phase current as an example, assuming that the trigger delay angle $\alpha=0$ and DC side inductance is infinite, 